\title{
ESTRATÉGIAS DE INTERVENÇÃO INTERDISCIPLINAR NO CUIDADO COM O PACIENTE COM TRANSTORNO ALIMENTAR: O TRATAMENTO FARMACOLÓGICO
}

\author{
STRATEGIES OF INTERDISCIPLINARY INTERVENTION IN THE CARE FOR \\ PATIENTS WITH EATING DISORDERS:PHRAMACOLOGICAL TREATMENT
}

Catalina Camas Cabrera

Médica Psiquiatra. Serviço de Interconsulta em Saúde Mental. Hospital das Clínicas da Faculdade de Medicina de Ribeirão Preto-USP Correspondência: Departamento de Neurologia, Psiquiatria e Psicologia Médica. Faculdade de Medicina de Ribeirão Preto-USP. Hospital das Clínicas de Ribeirão Preto. Av. Bandeirantes, 3900. CEP 14049-900 Ribeirão Preto, São Paulo, Brasil.

Fone: (016) 36022837 E-mail: catacabrera@terra.com.br

Cabrera CC. Estratégias de intervenção interdisciplinar no cuidado com o paciente com transtorno alimentar: o tratamento farmacológico. Medicina (Ribeirão Preto) 2006; 39 (3): 375-80.

RESUMO: Os Transtornos Alimentares são patologias psiquiátricas com apresentações distintas e critérios diagnósticos bem definidos, caracterizando-se por acentuada perturbação no modo de alimentar-se. Em função da gravidade da doença e comorbidades quase sempre associadas,o tratamento é longo e difícil para o paciente, família e equipe, exigindo adaptações a múltiplas formas de intervenção como atendimento ambulatorial, semi-internação ou até internação integral. Nesse sentido, as medicações psicotrópicas servem como coadjuvantes dentro do contexto da intervenção multiprofissional e interdisciplinar. De maneira geral, os pacientes com anorexia e bulimia nervosas resistem em tomar medicamento dessa natureza por temor associado ao ganho de peso e descrenças quanto à sua eficácia. Quase sempre é necessário negociar o uso da medicação e o sucesso da terapia vai depender da habilidade do médico em escolher o melhor momento para recomendá-la e do modo como este apresenta essa modalidade de intervenção. $\mathrm{Na}$ anorexia nervosa, o uso de psicotrópicos tem por objetivo auxiliar na recuperação do estado nutricional e atenuar os sintomas mentais que trazem muito desconforto. Já na bulimia nervosa, eles são indicados para reduzir os sintomas bulímicos e tratar as comorbidades psiquiátricas associadas. As medicações psicotrópicas comumente usadas são aqui apresentadas. Para garantir melhores resultados e sucesso no tratamento, a assistência ao paciente com transtorno alimentar deve ser realizada em serviços especializados, geralmente localizados em hospitais universitários de nível terciário, por profissionais capacitados que apresentam boa integração num trabalho em equipe.

Descritores: Transtornos da Alimentação. Anorexia Nervosa. Bulimia Nervosa. Tratamento.

\section{1- INTRODUÇÃO}

Os transtornos alimentares caracterizam-se por uma acentuada perturbação do comportamento alimentar. São condições patológicas relacionadas ao modo de alimentar-se.
As perturbações do comportamento alimentar desafiam nosso entendimento pois alimentar-se é, desde os primórdios, associado a sobrevivência, ao alívio de tensão (fome) e a satisfação (prazer).

No transtorno alimentar, por razões muito próprias, as pessoas experimentam ansiedades, medos ou 
culpas relacionadas a sua alimentação; idéias obsessivas associadas à imagem ideal de magreza e distorções na percepção da própria imagem corporal costumam levar a recusa deliberada de alimentos, apesar da fome, a ponto de por em risco a vida dessas pessoas, como acontece na anorexia nervosa. Por outro lado, uma pessoa com transtorno alimentar também pode, durante a noite num impulso, iniciar uma orgia alimentar numa refeição solitária e secreta, onde os alimentos são ingeridos rapidamente até o esgotamento do alimento ou da capacidade de ingestão, seguido de intenso desconforto físico e moral, como se percebe na bulimia nervosa.

Os transtornos alimentares são patologias psiquiátricas com apresentações distintas e critérios diagnósticos bem definidos. Aparecem predominantemente em mulheres $(90 \%)$ de todas as classes sociais, em faixa etária cada vez mais ampla (13-21, média 17 anos), com evolução fatal quando não tratados, em função da gravidade da doença ou das conseqüências desta. O tratamento dessas patologias é longo e difícil e vem motivando inúmeros estudos no Brasil e no mundo.

No Hospital das Clínicas da Faculdade de Ribeirão Preto-USP, começamos a adquirir maior confiança e sucesso nas intervenções quando passamos a estudar cada paciente e sua doença em grupo multiprofissional, buscando estabelecer um plano terapêutico personalizado, com múltiplas formas de intervenção associadas. Percebemos que a boa comunicação entre os profissionais que assistem a essas pessoas é fundamental, bem como reuniões periódicas do grupo, para troca de experiências e busca de diretrizes comuns, trabalhando juntos e integrados em uma equipe.

\section{2- ASPECTOS ESPECÍFICOS E IMPLICA- ÇÕES NO TRATAMENTO}

\section{a- Quanto a procura espontânea para tratamento}

Pacientes com anorexia nervosa não procuram espontaneamente ajuda; são levados ao médico contrariados e pouco dispostos a colaborar (até para fornecer informações). Desconfiam dos médicos que são vistos como inimigos interessados apenas em controlar sua alimentação e seu peso. Por não se sentirem doentes, todas as orientações médicas são rechaçadas. Em função disto esses profissionais precisam estar preparados para impasses e dificuldades na relação com o paciente.
Na bulimia nervosa o sofrimento faz com que o paciente procure ajuda, mas tal qual ocorre com pacientes com anorexia nervosa, existe uma dificuldade muito grande para aceitar o tratamento, expressa em oposição a quase todas as formas de intervenção propostas.

\section{b- Distorção da imagem corporal}

É uma alteração da sensopercepção (percepção de si mesmo) e está presente nas pessoas com anorexia nervosa, como critério diagnóstico inclusive. A percepção distorcida do corpo é a única distorção que essas pessoas têm da realidade, sendo que invariavelmente se sentem e se percebem mais gordos do que são.

Essa percepção distorcida não se modifica, quando o profissional utiliza técnicas de influenciação ou persuasão para "convencer" seu paciente da real imagem. Por isso, é sempre bom considerar se vale intervir neste aspecto diretamente, uma vez que este não é impeditivo para a adesão ao tratamento. Nesse sentido, um cuidado importante é o de não se colocar como objetivo do tratamento o ganho de peso.

A pessoa com bulimia pode ter distorção da imagem corporal, mas geralmente essa distorção aparece como indefinição quanto a se ver gordo ou magro - o corpo é percebido disforme.

\section{c- Ansiedade e medo de engordar}

A ansiedade relacionada ao ganho de peso é um sintoma presente na anorexia nervosa e na bulimia nervosa, mas tem características diferentes em cada uma delas: os pacientes com anorexia nervosa apresentam ansiedade intensa antes das refeições e evitam a alimentação em função dessa ansiedade, enquanto que os pacientes com bulimia nervosa sentem-se muito ansiosos após as refeições e por isso provocam as manobras purgativas (vômitos e diarréias) ou a hiperatividade (exercício físico).

Os pacientes com bulimia nervosa apresentam, além da ansiedade, intensos sentimentos de culpa por apresentarem um impulso descontrolado (compulsão), seguido de algum ritual de expurgo, situação habitualmente não aceita por eles mesmos.

\section{d- Auto-estima}

A auto-estima é baixa em todas as apresentações de transtorno alimentar, mas os pacientes com bulimia nervosa são os mais comprometidos sob este aspecto, a ponto de tentarem suicídio. 
Existem associações bem estabelecidas entre violência na infância (abandono, violência física ou abuso sexual) e anorexia nervosa na adolescência, bem como baixa auto-estima desde a infância e bulimia nervosa na adolescência ou vida adulta.

\section{e- Outros transtornos psiquiátricos associados}

Transtornos de personalidade e da impulsividade, transtornos de humor, transtornos de ansiedade e dependência de álcool e drogas podem estar associados aos transtornos alimentares (comorbidades), dificultando o tratamento e comprometendo o prognóstico.

Estas são condições que merecem acompanhamento psiquiátrico conjunto, tanto no tratamento ambulatorial quanto no tratamento hospitalar.

\section{3- LOCAIS DE TRATAMENTO}

Recomenda-se, para o sucesso do tratamento, que pacientes com transtornos alimentares sejam tratadas em serviços especializados.

No Brasil, os serviços especializados estão nos grandes centros, geralmente instalados em hospitais públicos de nível terciário, hospitais universitários ou em clínicas especializadas.

Os serviços especializados, com atendimento ambulatorial e leitos para internação, podem ser en- contrados em unidades de clínica médica, pediátrica e psiquiátrica.

A escolha da modalidade de tratamento (ambulatorial, semi-internação ou internação integral) dependerá da avaliação das condições clínicas, psiquiátricas e do apoio familiar para o tratamento do paciente. As indicações para o atendimento de pacientes com anorexia nervosa (AN) e bulimia nervosa (BN) são apresentadas no Quadro 1.

\section{4- EQUIPE}

A experiência tem demonstrado que a assistência ao paciente com transtorno alimentar requer cuidados especializados realizados por profissionais capacitados que atuam juntos, compondo uma equipe multiprofissional e interdisciplinar.

O trabalho em equipe interdisciplinar é mais eficaz que o tratamento clínico psicológico ou psiquiátrico exclusivo, é menos desgastante e atende às necessidades do paciente como um todo.

Os profissionais da equipe realizam as avaliações dos aspectos específicos relativos ao quadro mórbido, reúnem-se para firmar um diagnóstico "sistêmico", com diferentes abrangências (orgânico, situacional, familiar, etc...) e planejam a estratégia terapêutica.

\section{Quadro 1: Indicações para o atendimento de pacientes com anorexia nervosa (AN) e bulimia nervosa (BN)}

\section{AN}

Tratamento Ambulatorial

Possível desde que as reavaliações sejam freqüentes,

- Intervenção: paciente e família

- Programa estruturado
Semi-internação (HD) Pode ser indicado (Programa Estruturado) Indicações:
- Falha no tratamento ambulatorial
- Famílias muito disfuncionais
- Observação do comportamento e re- feições assistidas
Internação Integral
Pode ser necessário $(\mathrm{IMC}<16)$
Indicações:
- Falha no tratamento ambulatorial
- Para tratamento intensivo, onde a recupe- ração nutricional pode ser monito- rada (oral, enteral, parenteral),
- Correções das complicações orgânicas,
- Comorbidades psiquiátricas graves e tentativa de suicídio.

$\mathbf{B N}$

Habitual

Indicações:

- Acompanhamento regular

- Intervenção: paciente/família

Pouco sugerido e muito pouco acatado Indic ações:

- Complicações médicas graves,

- Transtornos psiquiátricos associados de difícil manejo (estabilização)

- Famílias muito disfuncionais

Pode ser necessário

Internações muito breves

Indic ações:

- Controle dos desequilíbrios metabólicos,

- Hematêmese,

- Ciclo bulimia/purgação incontroláveis,

- Comorbidades psiquiátricas graves e tentativa de suicídio. 


\section{5- ESTRATÉGIAS PARA O SUCESSO TERA- PÊUTICO}

\section{a- Quanto à equipe}

Espera-se que os profissionais possam desenvolver bom relacionamento interpessoal, confiança em cada colega e no grupo e que estejam sempre em sintonia "falando a mesma língua", pois pacientes com anorexia nervosa, por exemplo, quando são internados a contra gosto, se valem das tensões e contradições entre os profissionais que os assistem para sabotarem o próprio tratamento.

As reuniões constantes durante todo o tratamento (reuniões semanais ou quinzenais), são imprescindíveis para troca de experiências e avaliação das mesmas.

\section{b- Intervenções combinadas}

Tanto no tratamento ambulatorial como no hospitalar as intervenções combinadas são mais eficazes que as isoladas e são dirigidas ao paciente e família.

O restabelecimento do estado nutricional, associado as orientações alimentares, medicações psicotrópicas, terapia ocupacional e apoio psicológico fazem parte do programa estruturado para o paciente.

A família é motivada a freqüentar os grupos semanais de orientação aos familiares e a iniciar uma terapia familiar. Os serviços especializados dispõem de grupos de orientação nutricional e psicoterapia para pacientes e familiares.

\section{c- Planejamento terapêutico}

Recomenda-se firmar um contrato terapêutico entre equipe, paciente e família, com metas estabelecidas para a recuperação do estado nutricional, ritmo de recuperação, regras da instituição quanto à adesão ao tratamento ambulatorial e critérios para internação integral.

Uma política de "acordos mínimos" deve permear todo o tratamento, seja ele ambulatorial ou hospitalar.

As avaliações da evolução do tratamento, realizadas tanto entre os membros da equipe entre si, quanto entre a equipe, o paciente e sua família, devem ser regulares, bem como devem ser claros os critérios que definem as condições de alta, seja o tratamento ambulatorial ou hospitalar.

\section{6- INDICAÇÕES FARMACOLÓGICAS}

As medicações psicotrópicas devem ser vistas como coadjuvantes no tratamento dos transtornos alimentares e dentro do contexto da intervenção multiprofissional.

De um modo geral, pacientes com anorexia nervosa e bulimia nervosa não aceitam tomar medicações, colocando como justificativas temores associados a ganho de peso e descrenças quanto à eficácia das mesmas. Tentam habitualmente negociar o uso de medicações, lêem as bulas, questionam os médicos sobre a incidência dos efeitos colaterais e geralmente referem tais efeitos com pouco tempo de uso das mesmas.

O sucesso na instalação da terapia medicamentosa depende: da habilidade do médico em escolher o melhor momento de recomendá-la (ou seja, depois de instalada uma relação de confiança) e do modo como este profissional apresenta essa modalidade de intervenção, (com objetividade, sem supervalorização dos efeitos benéficos e nem desvalorização dos efeitos adversos).

Se decidir usar psicofármacos, o profissional deve dar preferência para as medicações que está familiarizado, mostrar-se flexível para adaptações pessoais mas firme no propósito de manutenção por um tempo suficiente para reposta terapêutica.

\section{a- Anorexia nervosa}

O uso de psicotrópicos tem por objetivo auxiliar na recuperação do estado nutricional e atenuar sintomas mentais que trazem muito desconforto.

\section{Ciproerptadina (Periatin $\left.{ }^{\circledR}\right)$}

É um antihistamínico e antagonista da serotonina com forte efeito orexígeno, além de apresentar efeito sedante. É recomendado nos quadros de anorexia nervosa grave do tipo restritivo, principalmente quando existe hiperatividade associada. As evidências têm mostrado que o benefício não se mantém a longo prazo, mas pode ser uma opção interessante para os primeiros dias de tratamento hospitalar.

\section{Clorpromazina (Amplictil®)}

É um antipsicótico com potente efeito antiemético. Em baixas doses ( $20 \mathrm{a} 75 \mathrm{mg} / \mathrm{dia}$ ) pode reduzir a freqüência de vômitos na anorexia nervosa do tipo purgativo, melhora a aceitação de alimentos, o sono e 
diminui a hiperatividade. Observa-se em muitos casos, a melhora das preocupações obsessivas, do funcionamento cognitivo e da adaptação social.

Recomenda-se iniciar essa medicação sob assistência médica (internação ou seminternação) em função da possibilidade de se monitorar os efeitos adversos (hipotensão arterial e sedação), caso surjam.

\section{Cloridrato de metoclopramida (Plasil $®$ )}

Pode ser usado para melhorar a sensação de plenitude gástrica, sintoma comum em anoréticos graves. É utilizado no tratamento hospitalar na dose de 10mg, 10 minutos antes das refeições, nos primeiros dias de internação. Com a recuperação nutricional o sintoma tende a desaparecer e a necessidade de tal medicação também.

\section{Antidepressivos}

Não são recomendados no início do tratamento, pois muitos dos sintomas associados a depressão (apatia, desinteresse, anedonia, insônia) podem desaparecer com a reposição nutricional. Na fase de manutenção, caso persistam sintomas que preencham os critérios para um episódio depressivo ou mesmo um transtorno depressivo, recomenda-se tratamento medicamentoso. As medicações mais usadas são:

Fluoxetina: na dose de 10 a 60mg/dia (convém estar atento ao uso abusivo, pois muitas pacientes esperam diminuir a fome com a medicação) e os outros inibidores seletivos da recaptação de serotonina (ISRS) como citaloprano, na dose de 10 a $20 \mathrm{mg} / \mathrm{dia}$.

Imipramina: na dose de 75 a 120mg/dia (observar retenções líquidas e aumento de peso que são motivos para o abandono do tratamento).

Ansiolíticos: recomendados para a ansiedade relacionada às refeições, são administrados meia hora antes do almoço e do jantar.

Bromazepan (Lexotan $®)-1.5 \mathrm{mg}$

Clonazepan (Rivotril@) $-1 \mathrm{mg}$

Lorazepan (Lorax $\left.{ }^{\circledR}\right)-0.5$ a $1 \mathrm{mg}$

Não é recomendado o uso continuado devido ao risco de dependência e habituação.

\section{b- Bulimia nervosa}

Os psicofármacos são indicados e eficazes para reduzir os sintomas bulímicos e para tratar as comorbidades psiquiátricas associadas, embora muitos pacientes expressem resistência ao uso ou fazem uso de modo inapropriado.

\section{Antidepressivos}

Auxiliam na redução da compulsão e dos vômitos. Os antidepressivos tricíclicos ainda são muito usados, como a Imipramina $50-125 \mathrm{mg} / \mathrm{dia}$ (Tofranil®) e Clorimipramina 75-125 mg/dia. Os inibidores seletivos da serotonina (ISRS), são bastante seguros e relativamente bem tolerados, em tratamentos de 6 meses a 1 ano.

Fluoxetina (Daforin ${ }^{\circledR}$, Fluxene $\AA$, Prozac $\AA$, Verotina $\left.{ }^{\circledR}\right)-60$ a $80 \mathrm{mg} / \mathrm{dia}$

Sertralina (Tolrest ${ }^{\circledR}$, Zoloft $\left.{ }^{\circledR}\right)-50$ a 200 mg/ dia.

Citalopram $\left(\left(\right.\right.$ Citta $^{\circledR}$, Cipramil® ${ }^{\circledR}$, Deriel ${ }^{\circledR}$, Proximax ${ }^{\circledR)} 20$ a 60 mg/dia.

Se existir um transtorno depressivo associado, o tratamento com essas medicações deve estenderse além de um ano. O sucesso do tratamento é maior quando se associa aconselhamento nutricional, psicoterapia e psicofarmacoterapia.

Ansiolíticos: não são muito recomendados em função do alto risco de dependência, associação com álcool e drogas. Cautela com a quantidade de medicamentos prescritos devido ao risco de suicídio por ingestão (overdose).

Estimulantes: em estudo Metilfenidato (Ritalina $\left.{ }^{\circledR}\right)$ para pacientes com transtorno de impulso associado, refratárias ao tratamento convencional.

\section{7- PROGNÓSTICO COM TRATAMENTO}

\section{a- Anorexia nervosa}

As taxas de melhora e cura encontradas, para pacientes que seguiram um tratamento regular são: $40 \%$ recuperam-se totalmente; $30 \%$ melhoram sensivelmente; $20 \%$ cronificam; $10 \%$ morrem. De um modo geral o prognóstico é pior quando existe comorbidade, como por exemplo, transtorno de personalidade boderline, evitativo, narcísico e pacientes que demoram a iniciar o tratamento.

\section{b- Bulimia nervosa}

De um modo geral $30 \%$ apresentam remissão total dos sintomas; $50 \%$ melhoram os sintomas, mas apresentam recaídas e $20 \%$ mantêm-se doentes. O prognóstico é pior quando existe associação com alcoolismo, abuso-dependência de drogas; transtorno boderline e do impulso. 
Cabrera CC. Strategies of interdisciplinary intervention in the care for patients with eating disorders: pharmacological treatment. Medicina (Ribeirão Preto) 2006; 39 (3): 375-80.

ABSTRACT: Eating disorders are psychiatric diseases with distinct presentations and well defined diagnostic criteria, characterized by marked perturbation in eating mode. In view of the severity of the disease an of the co-morbidities almost always associated, treatment is long and difficult for the patient, the family and the team, requiring adaptation to multiple forms of intervention such as ambulatory care, semi-hospitalization or even full hospitalization. In this respect, psychotropic medications serve as adjuvant within the context of multiprofessional and multidisciplinary intervention. In general, patients with anorexia and bulimia nervosa resist treatment with these medications because of their fear to gain weight and a disbelief in their efficacy. It is almost always necessary to negotiate the use of medication and the success of treatment depends on the ability of the doctor to choose the best time to recommend it and on how the doctor presents this modality of intervention. In anorexia nervosa, the objective of the use of psychotropic drugs is to aid the recovery of nutritional status and to reduce the mental symptoms that cause great discomfort. In bulimia nervosa, these medications are indicated in order to reduce the bulimic symptoms and to treat the associated psychiatric co-morbidities. The psychotropic medications commonly used are presented here. To guarantee better results and a successful treatment, patients with eating disorders should be treated in specialized services, usually located in tertiary care university hospitals, by qualified professionals who integrate well with team work.

Keywords: Eating Disorders. Anorexia Nervosa. Bulimia Nervosa. Treatment.

\section{LEITURAS RECOMENDADAS}

1 - Agnas WS, Rossiter EM, Arnow B, Scheider JÁ, Telch CF, Rocebur SD, Bruce B, Perl M, Koran LM. Pharmacologic and cognitive-behavioral treatment for bulimia nervosa. A controlled comparison. Am J Psychiatry 1992, 149:82-7.

2 - Azevedo MA, Guerra VNA, orgs. Infância e violência doméstica: fronteiras do conhecimento. São Paulo; Cortez, 2000.

3 - Bulick CM, Prescott CA, Kendler KS.Features of childhood sexual abuse and the development of psychiatric and substance use disorders. Br J Psychiatry 2001; 179:444-9.

4 - Claudino AM, Jorge MR. Diagnóstico e conduta na bulimia nervosa. In: Mari JJ, Razzouk D, Peres MFT, Porto JÁ, coords. Guia de Psiquiatria. Barueri: Ed. Manole; São Paulo: Escola Paulista de Medicina - UNIFESP; 2002. p. 110-20.

5 - Cordás IA. Bulimia nervosa: diagnóstico e propostas de tratamento. São Paulo: Editora Lemos, 1998.

6 - Fairburn CG. A prospective study of outcome in bulimia nervosa and the longterm effects of three psychological treatments. Arch Gen Psychiatry 1995; 52:304-8.

7 - Freitas S, Lopes CS, Coutinho W, Apolinário JC. Tradução e adaptação para o português da Escala de Compulsão Alimentar Periódica. Rev Bras Psiquiatr 2001; 23(4): 251-20.

8 - Harriet M, Fleming JE. Childhood abuse and lifetime psychopatology in a community sample. Am J Psychiatry 2001; 158(11):1878-83.

9 - Herscovici CR, Bay L. Anorexia nervosa e bulimia - ameaças à autonomia. Porto Alegre: Artes Médicas, 1997.
10 - Kaplan HI, Sadock BJ. Manual de Psiquiatria clínica, $2^{\text {a }}$. ed. Porto Alegre: Artes Médicas, 1998.

11 - Laliberté M, Bolond FJ, Leichner P.Family Climates: family factors especific to disturded eating and bulimia nervosa. J Clin Psychol 1999, 55(9):1021-40.

12 - Matos MIR, Aranha LS, Faria AN, Ferreira SRG, Bacaltchuck $\mathrm{J}$, Zanella MT. Binge eating disorder, anxiety depression and body image in grade III obesidy patients. Rev Bras Psiquiatr 2002; 24(4): 165-9.

13 - Morgan CM, Negrão AB. Diagnóstico e conduta na anorexia nervosa. In: Mari JJ, Razzouk D, Peres MFT, Porto JÁ, coords. Guia de Psiquiatria. Barueri: Ed. Manole; São Paulo: Escola Paulista de Medicina - UNIFESP; 2002. p. 99-110.

14 - Sadock BJ, Sadock VA. Manual de farmacologia psiquiátrica de Kaplan e Sadock, $3^{\mathrm{a}}$. ed. Porto Alegre: Artmed; 2002.

15 - Sokol MS, Gray NS, Goldstein A, Kaye WH. Methylphenidate treatment for bulimia nervosa associated with a cluster B personality disorder. Int J Eat Disord 1999; 25(2):233-7.

16 - Stéfano SC, Borges MBF, Claudino AM. Transtorno da compulsão alimentar periódica. In: Mari JJ, Razzouk D, Peres MFT, Porto JÁ, coords. Guia de Psiquiatria. Barueri: Ed. Manole; São Paulo: Escola Paulista de Medicina - UNIFESP; 2002. p 121-5.

17 - Vitielo B, Lederhendler I. Research of eating disorders: current status and future proaspects. Biol Psychiatry 2000; 47, 777-86. 